\title{
COMBINED LOPES AND KASCADE-GRANDE DATA ANALYSIS
}

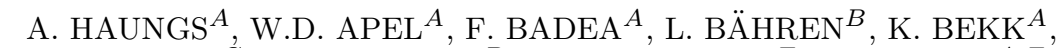
A. BERCUCI $^{C}$, M. BERTAINA ${ }^{D}$, P.L. BIERMANN ${ }^{E}$, J. BLÜMER ${ }^{A, F}$,

H. BOZDOG ${ }^{A}$, I.M. BRANCUS ${ }^{C}$, M. BRÜGGEMANN ${ }^{G}$, P. BUCHHOLZ $^{G}$, S. BUITINK ${ }^{H}$, H. BUTCHER ${ }^{B}$, A. CHIAVASSA ${ }^{D}$, K. DAUMILLER $^{A}$, A.G. DE BRUYN ${ }^{B}$, C.M. DE $\operatorname{VOS}^{B}$, F. DI PIERRO ${ }^{D}$, P. DOLL ${ }^{A}$,

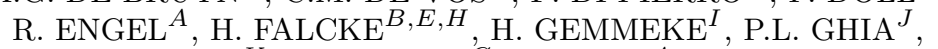
R. GLASSTETTER ${ }^{K}$, C. GRUPEN ${ }^{G}$, D. HECK $^{A}$, J.R. HÖRANDEL ${ }^{F}$, A. HORNEFFER ${ }^{H, E}$, T. HUEGE $^{A, E}$, K.-H. KAMPERT ${ }^{K}$, G.W. KANT ${ }^{B}$,

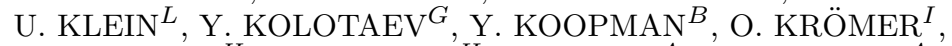
J. KUIJPERS ${ }^{H}$, S. LAFEBRE ${ }^{H}$, G. MAIER ${ }^{A}$, H.J. MATHES ${ }^{A}$, H.J. MAYER ${ }^{A}$, J. MILKE ${ }^{A}$, B. MITRICA ${ }^{C}$, C. MORELLO ${ }^{J}$, G. NAVARRA ${ }^{D}$, S. NEHLS ${ }^{A}$, A. NIGL $^{H}$, R. OBENLAND ${ }^{A}$, J. OEHLSCHLÄGER ${ }^{A}$, S. OSTAPCHENKO ${ }^{A}$, S. OVER ${ }^{G}$, H.J. PEPPING ${ }^{B}$, M. PETCU ${ }^{C}$, J. PETROVIC ${ }^{H}$, T. PIEROG ${ }^{A}$, S. PLEWNIA ${ }^{A}$, H. REBEL $^{A}$, A. $\operatorname{RISSE}^{M}$, M. ROTH $^{F}$, H. SCHIELER ${ }^{A}$, G. SCHOONDERBEEK ${ }^{B}$,

O. SIMA ${ }^{C}$, M. STÜMPERT ${ }^{F}$, G. TOMA ${ }^{C}$, G.C. TRINCHERO ${ }^{J}$,

H. ULRICH ${ }^{A}$, J. VAN BUREN ${ }^{A}$, W. VAN CAPELLEN ${ }^{B}$,

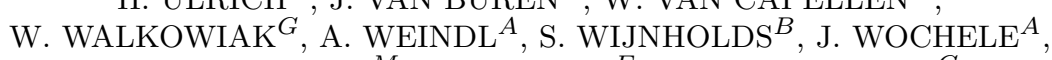
J. ZABIEROWSKI ${ }^{M}$, J.A. ZENSUS ${ }^{E}$, D. ZIMMERMANN ${ }^{G}$ LOPES COLLABORATION

${ }^{A}$ Institut für Kernphysik, Forschungszentrum Karlsruhe, Germany $B$ ASTRON Dwingeloo, The Netherlands C NIPNE Bucharest, Romania

D Dpt di Fisica Generale dell'Università Torino, Italy

E Max-Planck-Institut für Radioastronomie, Bonn, Germany

$F$ Institut für Experimentelle Kernphysik, Uni Karlsruhe, Germany,

$G$ Fachbereich Physik, Universität Siegen, Germany

${ }^{H}$ Dpt of Astrophysics, Radboud Uni Nijmegen, The Netherlands

I IPE, Forschungszentrum Karlsruhe, Germany

${ }^{J}$ Ist di Fisica dello Spazio Interplanetario INAF, Torino, Italy

K Fachbereich Physik, Uni Wuppertal, Germany

${ }^{L}$ Radioastronomisches Institut der Uni Bonn, Germany

${ }^{M}$ Soltan Institute for Nuclear Studies, Lodz, Poland 
First analyses of coincident data of the LOPES (LOfar PrototypE Station) radio antennas with the particle air shower experiment KASCADE-Grande show basic correlations in the observed shower parameters, like the strength of the radio signal and the particle number, or comparing the estimated shower directions. In addition, an improvement of the experimental resolution of the shower parameters reconstructed by KASCADE-Grande can be obtained by including the data of the radio antennas. This important feature will be shown in this article explicitely by an example event.

The KASCADE ${ }^{1}$ (KArlsruhe Shower Core and Array DEtector) experiment measures showers in a primary energy range from $100 \mathrm{TeV}$ to $80 \mathrm{PeV}$ and provides multi-parameter measurements on a large number of observables concerning electrons, muons at 4 different energy thresholds, and hadrons. The main detector components of KASCADE are a field array, the so called central detector and a muon tracking detector. The field array consists of 252 detector stations with shielded as well as unshielded scintillation detectors for measuring the electromagnetic and the muonic shower component.

KASCADE-Grande ${ }^{2}$ is the extension of the multi-detector setup KASCADE to cover a primary cosmic ray energy range from $100 \mathrm{TeV}$ to $1 \mathrm{EeV}$. Grande is an array of $700 \times 700 \mathrm{~m}^{2}$ equipped with 37 plastic scintillator stations sensitive to measure energy deposits and arrival times of air shower particles.

At present, LOPES ${ }^{3}$ operates 30 dipole radio antennas (LOPES-30) positioned inside or nearby KASCADE. For the present analysis only data of LOPES-10 (10 antennas in operation), which was running for 5 months, is used. The antennas operate in the frequency range of 40-80 MHz. The radio data is collected when a "large event" trigger is received from KASCADE which translates to primary energies above $10^{16} \mathrm{eV}$.

The LOPES-10 data set is subject of various analyses using different selections: With an event sample obtained by hard cuts the proof of principle to detect air showers in the radio frequency range was given ${ }^{3}$. With events fallen inside KASCADE the basic correlations with shower parameters are shown ${ }^{4}$. Further interesting features are investigated with a sample of very inclined showers ${ }^{5}$ and with a sample of events measured during thunderstorms ${ }^{6}$.

Here we report results from an analysis performed by correlating the radio signals measured by LOPES-10 with EAS events reconstructed by KASCADE-Grande with remote cores included ${ }^{7}$. Grande is taking data 
in coincidence with KASCADE and LOPES and enables to reconstruct showers with primary energies up to $10^{18} \mathrm{eV}$ and with distances between shower core and the LOPES-10 antennas up to $700 \mathrm{~m}$. The Grande reconstruction accuracy of shower core position and direction is in the order of $4 \mathrm{~m}(13 \mathrm{~m})$ and $0.18^{\circ}\left(0.32^{\circ}\right)$ with $68 \%(95 \%)$ confidence level for simulated proton and iron showers at $100 \mathrm{PeV}$ primary energy and $22^{\circ}$ zenith angle ${ }^{8}$.

A crucial element of the detection method is the digital beamforming which allows to place a narrow antenna beam in the direction of the cosmic ray event. This is possible because the phase information of the radio waves is preserved by the digital receiver and the cosmic ray produces a coherent pulse. This method is also very effective in suppressing interference from the particle detectors which radiate incoherently. The procedure of time shifting of the radio signals in the antennas is relatively safe when based on the values provided by the reconstruction for shower core and shower axis using the data of the original KASCADE field array. Due to the high granularity of the detector stations the accuracy of core and direction reconstruction is high enough to obtain a good coherence of the radio signals. The shower reconstruction using information from the Grande array is required for shower cores outside KASCADE. The Grande stations, $10 \mathrm{~m}^{2}$ of plastic scintillator detectors each, are spaced at $\simeq 130 \mathrm{~m}$ and cannot assure an accuracy comparable with the original KASCADE array. So, a so-called 'optimised' beamforming is performed, which searches for a maximum coherence by varying the core and the direction around the values provided by the Grande reconstruction.

In five months of LOPES-10 data taking a sample of 862 candidate events were selected. Selection criteria were i) coincident measurement of the event by LOPES 10, KASCADE field array which have triggered LOPES, and Grande array; ii) zenith angle of the shower less than $50^{\circ}$; iii) a geometrical cut that the core position lies inside the Grande array $\left(0.358 \mathrm{~km}^{2}\right)$; iv) to reduce the data sample additionally an energy and distance cut is applied which is motivated by Allan's fomula ${ }^{9}: \epsilon_{\nu}=$ $20 \cdot\left(\frac{E}{10^{17} \mathrm{eV}}\right) \cdot \sin \alpha \cdot \cos \theta \cdot \exp \left(-\frac{R}{R_{0}(\nu, \theta)}\right)\left[\frac{\mu V}{m \cdot M H z}\right]$. The formula describes the pulse amplitude per unit bandwidth $\left(\epsilon_{\nu}\right)$ of the radio signal induced by an EAS. Here $E$ is the primary energy, $\alpha$ the angle to the geomagnetic field, $\theta$ the zenith angle, $R$ the distance to the shower axis and $R_{0}$ the scaling radius $R_{0}=110 \mathrm{~m}$ at $55 \mathrm{MHz}$; the exponential radial factor plays a significant role for remote showers. The cut has been considered as 


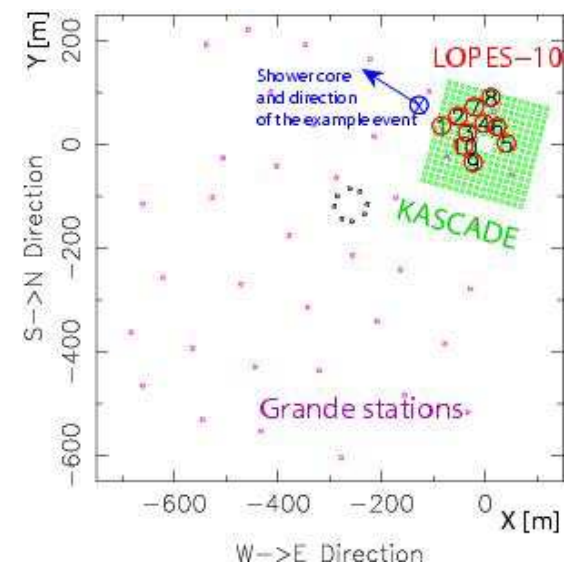

Figure 1. Sketch of the LOPES 10 layout inside KASCADE-Grande. Core position and shower direction of the candidate event 444 is also shown.

$\lg \left(\frac{E}{e V}\right)>\lg \left(\frac{E_{0}}{e V}\right)+0.4343 \cdot \frac{R}{R_{0}} \quad$ OR $\quad \lg \left(\frac{E}{e V}\right)>17.5 \quad$ with $E_{0}=10^{16.5} \mathrm{eV}$ and $R_{0}=160 \mathrm{~m}$, i.e. weaker than Allan's scaling with radius.

Fig. 1 shows the layout of LOPES-10 in KASCADE-Grande including the core position and direction of an event (number 444 of 862) with a clear radio signal. Fig. 2 shows the radio signal of the event before and after the optimized beamforming. An noticeable increase is seen in the final beam signal after the optimised beamforming. Table 1 contains the values (azimuthal angle $\phi$, zenith angle $\theta, X_{\text {core }}$ and $Y_{\text {core }}$ ) reconstructed by Grande and the corresponding values obtained after maximizing the radio coherence for this event; the small shifts assure an almost perfect coherence of the radio signal (lower left panel in Fig. 2).

Table 1. Shower parameters from the Grande reconstruction and after maximized radio coherence for an example event (geomagnetic angle $\alpha=57.9^{\circ}$, $\lg (\mathrm{E} / \mathrm{eV})=17.73)$.

\begin{tabular}{|c|c|c|}
\hline parameter & Grande reconstruction & maximized radio coherence \\
\hline$\phi$ & $302.2^{\circ}$ & $299.3^{\circ}$ \\
$\theta$ & $41.0^{\circ}$ & $39.9^{\circ}$ \\
$X_{\text {core }} / \mathrm{m}$ & -142.8 & -139.2 \\
$Y_{\text {core }} / \mathrm{m}$ & 40.3 & 51.6 \\
curvature $/ \mathrm{m}$ & 3250 & 4250 \\
\hline
\end{tabular}

The maximization of the radio coherence (optimised beamforming) will be performed to all the candidate events. This will not only increase the 

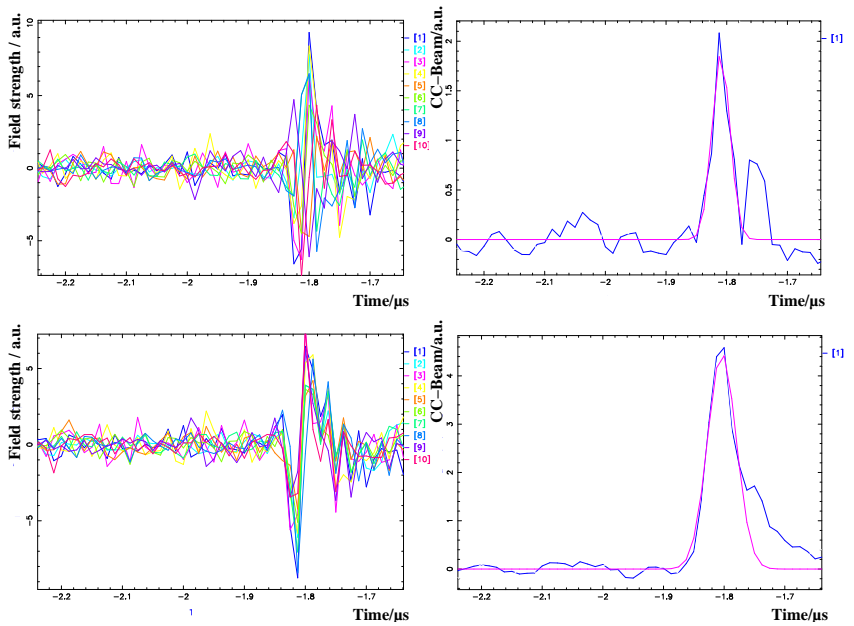

Figure 2. Radio signals in the 10 antennas and the resulting radio signal (so-called cross-correlation beam) with a gaussian fit to the signal after first beamforming (upper panels) and after optimised beamforming (lower panels).

efficiency $(\approx 30 \%$ without optimized beamforming) for finding radio signals of air showers, but also improve the reconstruction quality on the primary energy of the cosmic rays if the information is included in the shower reconstruction of Grande.

\section{References}

1. T. Antoni et al. - KASCADE collab., Nucl. Instr. $\mathcal{G}$ Meth. A 513, 429 (2003).

2. G. Navarra et al. - KASCADE-Grande collab., Nucl. Instr. \& Meth. A 518, 207 (2004).

3. H. Falcke et al. - LOPES collab., Nature 435, 313 (2005).

4. A. Horneffer et al. - LOPES collab., Detection of radio pulses from extensive air showers, Proc. of $29^{\text {th }}$ ICRC, Pune, India (2005).

5. J. Petrovic et al. - LOPES collab., Radio emission of highly inclined cosmic ray air showers measured with LOPES, Proc. of $29^{\text {th }}$ ICRC, Pune, India (2005).

6. S. Buitink et al. - LOPES collab., Electric field influence on the radio emission of air showers, Proc. of $29^{\text {th }}$ ICRC, Pune, India (2005);

7. A.F. Badea et al. LOPES collab., Remote event analyses of LOPES-10, Proc. of $29^{\text {th }}$ ICRC, Pune, India (2005);

A.F. Badea et al. LOPES collab., First determination of the reconstruction resolution of an EAS radio detector, Proc. of $29^{\text {th }}$ ICRC, Pune, India (2005);

8. R. Glasstetter et al. - KASCADE-Grande collab., Proc. of $28^{\text {th }}$ ICRC, Tsukuba, Japan, 781 (2003).

9. H.R. Allan, Prog. in Element. Part. and Cos. Ray Phys., 10, 171 (1971). 